Proceeding Series of the Brazilian Society of Computational and Applied Mathematics

\title{
Sincronismo entre Redes Neurais Complexas: Um Modelo de Sistema Visual de Mamíferos
}

\author{
Brian Lee Mayer ${ }^{1}$ \\ Programa de Pós-graduação em Engenharia Elétrica e Computação da Universidade \\ Presbiteriana Mackenzie, São Paulo, SP \\ Luiz Henrique Alves Monteiro ${ }^{2}$ \\ Escola de Engenharia da Universidade Presbiteriana Mackenzie e Escola Politécnica da \\ Universidade de São Paulo, São Paulo, SP
}

\begin{abstract}
Resumo. Aqui, usam-se redes do tipo Newman-Watts num modelo das vias neurais do sistema visual de mamíferos. Essas vias são encontradas em cada hemisfério cerebral e se comunicam por meio do corpo caloso. O objetivo é investigar, numericamente, a ocorrência de atividade neural síncrona nessa rede complexa mediante estímulo periódico.
\end{abstract}

Palavras-chave. Neurodinâmica, rede complexa, sincronismo, via neural, visão.

\section{Introdução}

Simplistamente, redes complexas podem ser definidas como grafos aleatórios com topologia de acoplamento não trivial [7]. Redes neurais biológicas são exemplos típicos. Um modelo interessante de rede complexa é aquele que foi proposto por Mark E. J. Newman e Duncan J. Watts, em 1999 [8]. A rede de Newman-Watts é construída a partir de uma estrutura regular, na qual se adicionam conexões aleatórias entre seus nós. Esse procedimento resulta num grafo com características topológicas típicas de redes mundopequeno (que são menor caminho médio proporcional ao logaritmo do número de nós e coeficiente de agregação da ordem de um [7]). Nos trabalhos teóricos e/ou numéricos em que essa topologia é empregada como um modelo de rede neural biológica, cada nó corresponde a um neurônio, cuja atividade é função do estímulo recebido [6,9]. Esse estímulo pode advir de neurônios vizinhos ou pode ter origem externa à rede. Com o passar do tempo, alguns neurônios podem sincronizar seus disparos. De fato, em diversos grafos do mundo real, os nós constituintes possuem dinâmica própria, como os

$171462287 @$ mackenzista.com.br

2 luizm@mackenzie.br, luizm@usp.br 
neurônios de um cérebro, e em tais grafos, pode ser que uma parte dos nós sincronize suas atividades, ao menos durante certo intervalo de tempo [10].

Em neurociência, estudos sobre sincronismo são bastante relevantes, pois sincronismo parece ser crucial para a correta execução de funções cognitivas e sensoriais que dependem da interação coordenada de neurônios localizados em regiões distintas do cérebro [2]. Por exemplo, medidas experimentais relacionam segmentação e compreensão de imagens à sincronização de disparos de neurônios ao longo das vias neurais do sistema visual de mamíferos [2,6]. Entretanto, desordens neurológicas, como autismo, epilepsia, esquizofrenia e mal de Parkinson, estão correlacionadas a níveis aumentados de atividade síncrona [7,11]. Grosso modo, em redes neurais biológicas, sincronismo em pequena escala é saudável; em larga escala, é patológico. Percebe-se, portanto, que estudos sobre comportamento síncrono em redes neurais podem auxiliar na compreensão do funcionamento normal do cérebro, o que pode ainda sugerir a criação de máquinas bioinspiradas ou levar à proposição de novos algoritmos de computação natural. Tais estudos também podem ser importantes em investigações sobre neuropatologias $[7,11]$.

Atividade síncrona é detectada em neurônios da retina, do núcleo geniculado lateral (do tálamo) e do córtex cerebral de mamífero, após estímulo visual [3-5]. Monteiro e Martins investigaram a ocorrência de sincronismo nessa via neural, usando um modelo baseado em autômatos celulares e redes complexas [6]. Aqui, investiga-se a dinâmica de duas vias neurais acopladas. Sabe-se que a via presente no hemisfério cerebral esquerdo acopla-se àquela do hemisfério direito por meio do corpo caloso [1]. Nesse nosso modelo, cada via neural é modelada por uma rede complexa do tipo Newman-Watts, que recebe uma entrada externa periódica.

Este artigo sobre sincronismo entre redes complexas está assim organizado. $\mathrm{Na}$ Seção 2, descrevem-se o modelo de rede e o modelo de neurônio usados neste trabalho, e define-se sincronismo. Na Seção 3, apresentam-se os resultados obtidos por simulações numéricas. Esses resultados são discutidos na Seção 4.

\section{O modelo e a definição de sincronismo}

Considere a via neural formada por retina, núcleo geniculado lateral e córtex visual. O núcleo geniculado e o córtex são representados por reticulados regulares, cada qual com dimensões $n \times n$, em que cada nó se acopla aos seus vizinhos situados a norte, a sul, a leste e a oeste (os nós da borda possuem, obviamente, menos vizinhos). Além dessas conexões regulares, há $s$ intraconexões aleatórias em cada reticulado. O acoplamento entre cada par de reticulados, ao longo da mesma via neural, se dá por meio de $p$ interconexões aleatórias. Estas são estabelecidas entre as linhas $l_{1}$ e $l_{2}$ do reticulado do núcleo e as linhas $l_{3}$ e $l_{4}$ do reticulado do córtex. As duas vias conectam-se a um reticulado regular de dimensões $m \times m$, que faz o papel do corpo caloso. Este se acopla a cada uma das vias por $q$ conexões aleatórias, estabelecidas entre as linhas $l_{1}$ e $l_{2}$ do reticulado associado ao núcleo geniculado, e entre as linhas $l_{3}$ e $l_{4}$ do reticulado associado ao córtex. A Figura 1 mostra um grafo em que $n=10, m=4, s=0, p=10, q=6, l_{1}=9$, $l_{2}=10, l_{3}=1$ e $l_{4}=2$. A rede complexa assim construída é do tipo Newman-Watts [8]. 


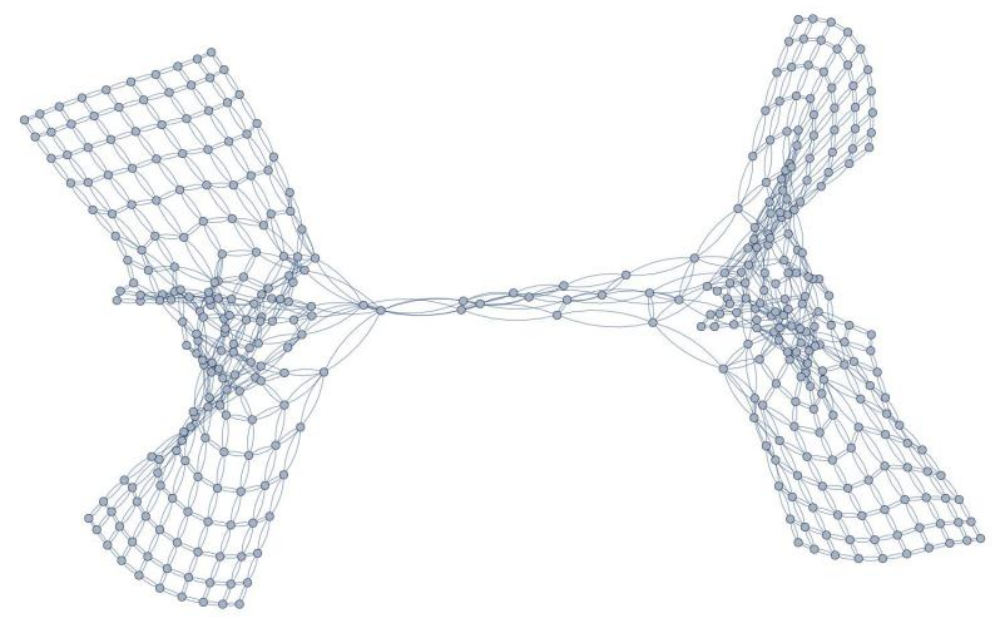

Figura 1: Grafo gerado considerando $n=10, m=4, s=0, p=10, q=6, l_{1}=9, l_{2}=10, l_{3}=1$ e $l_{4}=2$. Os reticulados do lado esquerdo correspondem à via neural do hemisfério esquerdo; aqueles do lado direito, à via do hemisfério direito. Os hemisférios estão interligados pelo reticulado central, que representa o corpo caloso.

Cada nó do grafo corresponde a um neurônio, que recebe estímulos dos neurônios a ele conectados ou o estímulo (de origem externa) associado à retina. A cada passo de tempo $t, \mathrm{o}$ neurônio encontra-se em um de três estados: ou ele está em repouso, ou ativo (disparando) ou refratário. As regras de transição de estados são [6]: um neurônio em repouso no passo de tempo $t$ dispara em $t+1$ se a soma dos valores dos estímulos recebidos é maior ou igual ao limiar $L$; um neurônio que disparou em $t$ passa para o estado refratário em $t+1$; um neurônio no estado refratário em $t$ passa para estado de repouso em $t+1$. Note que essas regras são deterministas. Ao disparar, o neurônio envia um sinal de magnitude $s$ aos seus vizinhos. Esse sinal é multiplicado pela intensidade da conexão sináptica $c$ e é o produto $s c$ que denota o estímulo advindo de um neurônio vizinho. Aqui, toma-se $s=1, c=1$ e $L=1$, de modo que um neurônio em repouso dispara em $t+1$ se ao menos um de seus vizinhos estiver ativo em $t$.

$\mathrm{O}$ reticulado referente ao núcleo geniculado lateral no hemisfério esquerdo é denotado por $i=1$; o reticulado referente ao córtex visual no hemisfério esquerdo por $i=2$; aquele associado ao núcleo geniculado lateral no hemisfério direito por $i=3$; aquele associado ao córtex visual no hemisfério direito por $i=4$; e o reticulado que representa o corpo caloso por $i=5$. Seja $x_{i}$ a fração de neurônios ativos no $i$-ésimo reticulado. Em tais reticulados, as conexões são não direcionadas (não dirigidas).

Suponha que, em regime permanente, $x_{i}$ oscile com período $T_{i}$. Aqui, diz-se que os reticulados $i$ e $j$ estão sincronizados se, em regime permanente, $T_{i} / k_{i}=T_{j} / k_{j}$ sendo $k_{i}$ e $k_{j}$ números inteiros. A intenção é verificar se as duas vias podem se sincronizar com o estímulo periódico vindo da retina. É sabido que a retina codifica estímulos visuais por meio de oscilações coerentes [3-5]. No nosso modelo, a retina equivale a uma entrada unitária periódica de período $T$, aplicada a um neurônio na primeira linha do(s) reticulado(s) $i=1$ e/ou $i=3$. Por exemplo, $T=3$ corresponde à sequência $100100100 \ldots$; ou seja, aplica-se uma entrada unitária a cada três passos de tempo. 


\section{Simulações numéricas}

A Figura 2 ilustra a evolução temporal da rede mostrada na Figura 1, para um estímulo de período dois aplicado apenas ao neurônio mais à esquerda da primeira linha do reticulado $i=1$. Note que, conforme o tempo passa, as frações $x_{i}$ de neurônios ativos nos reticulados $i=1,2,3,4$ convergem para uma oscilação de período quatro.
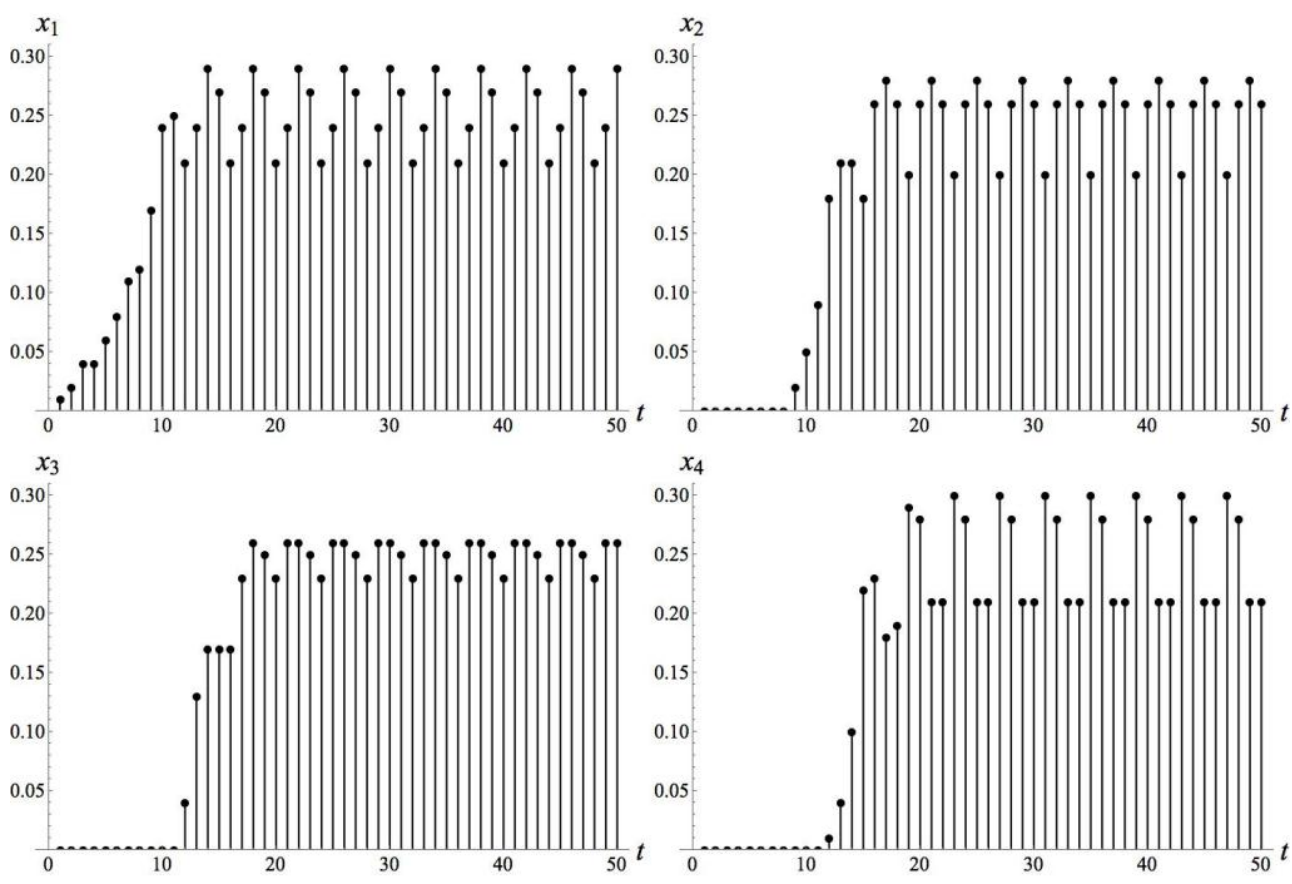

Figura 2: Porcentagem $x_{i}$ de neurônios ativos nos reticulados $i=1,2,3,4$ para um estímulo de período $T=2$ aplicado ao neurônio na primeira linha e na primeira coluna do reticulado $i=1$ da rede exibida na Figura 1. Com o passar do tempo, os quatros reticulados tendem a oscilar com período $T_{i}=4$. Observe que a magnitude de $x_{i}$ está em torno de $20 \%$.

A fim de investigar numericamente a dinâmica dessa rede complexa, foram realizadas as seguintes séries de simulações:

1. Um estímulo de período $T=2$ é aplicado apenas a um neurônio da primeira linha do reticulado $i=1$. Como há 10 neurônios na primeira linha, foram realizadas 10 simulações (de maneira que, em cada simulação, o estímulo foi aplicado a um neurônio diferente). Resultado: todos os quatro reticulados se sincronizaram com período $T_{i}=4(i=1,2,3,4)$, como mostrado na Figura 2 . Apesar de se obter $T_{i}=4$ em todas essas simulações, o padrão de ativação (isto é, a sequência de valores que cada $x_{i}$ exibe em regime permanente) foi diferente em cada simulação.

2. Idem ao anterior, com estímulo de período $T=3$. Resultado: os quatro reticulados se sincronizaram com período $T_{i}=3(i=1,2,3,4)$, com $x_{i}$ exibindo um padrão de ativação distinto em cada uma das 10 simulações e magnitude em torno de $30 \%$. 
3. Um estímulo de período $T=2$ é simultaneamente aplicado a um par de neurônios: um na primeira linha do reticulado $i=1$ e outro neurônio na primeira linha do reticulado $i=3$, de modo que ambos estejam na mesma coluna nos respectivos reticulados. Como há 10 pares de tais neurônios, foram realizadas 10 simulações. Resultado: todos os quatro reticulados se sincronizaram com período $T_{i}=4(i=1,2,3,4)$. A magnitude de $x_{i}$ permaneceu em torno de $20 \%$. Entretanto, a solução síncrona foi atingida mais rapidamente (cerca de metade do tempo, quando comparado com o caso em que o estímulo é aplicado a um único neurônio).

4. Idem ao anterior, com estímulo de período $T=3$. Resultado: os quatro reticulados se sincronizaram com $T_{i}=3(i=1,2,3,4)$. A magnitude de $x_{i}$ ficou em torno de $30 \%$ e o tempo necessário para se atingir o sincronismo foi em torno da metade, se comparado com as simulações em que apenas um neurônio é estimulado.

Essas quatro séries de simulações foram refeitas considerando que, agora, há $s=10$ intraconexões aleatórias em cada reticulado (ou seja, um total de 40 intraconexões aleatórias nas duas vias). Embora o padrão de ativação em regime permanente tenha se alterado e tal regime tenha sido atingido de forma mais rápida, o período da solução atratora não mudou: para $T=2$, os quatro reticulados se sincronizam com $T_{i}=4$; para $T=3$, então $T_{i}=3$. As magnitudes de $x_{i}$ também não mudaram de forma significativa, se comparadas ao caso $s=0$.

\section{Conclusões}

Aqui, investigou-se numericamente a dinâmica de redes do tipo Newman-Watts acopladas, sujeitas a entrada periódica. Tais redes foram usadas como um modelo das vias neurais associadas à percepção de estímulos visuais por mamíferos. Em cada hemisfério cerebral, há uma via (representada por dois reticulados $n \times n$ interligados), que se acopla com a outra via por meio do corpo caloso (representado pelo reticulado $m \times m$ ). Verificou-se que as vias tendem a se sincronizar; entretanto, o período de oscilação varia com o período do estímulo externo (que corresponde ao sinal enviado pela retina). No caso de estímulo com $T=2$, as vias tendem a uma oscilação com $T_{i}=2 T$; no caso em que $T=3$, as vias tendem a oscilar com $T_{i}=T$. É interessante salientar que medidas experimentais em mamíferos revelam que retina, núcleo geniculado lateral e córtex visual tendem a se sincronizar na mesma frequência ou numa frequência que é a metade da frequência do estímulo aplicado [3-6], o que está de acordo com os resultados do nosso modelo simplista. A inclusão de intraconexões aleatórias e/ou a aplicação simultânea do estímulo nas duas vias diminuíram o tempo de sincronização, mas não alteraram substancialmente os valores de $x_{i}$. Continuações necessárias para este trabalho são:

1. Selecionar os valores dos parâmetros $n, m, s, p, q, l_{1}, l_{2}, l_{3}, l_{4}$ (das redes) e $T$ (do estímulo) de modo que o modelo represente mais fielmente as vias neurais investigadas. Este trabalho e aquele realizado por Martins e Monteiro [6] (para uma única via) sugerem que temos um ponto de partida promissor.

2. Incluir sinapses inibitórias (nas duas vias e no corpo caloso) e analisar seus efeitos sobre a atividade síncrona inter-hemisférica [1]. 


\section{Agradecimentos}

Brian L. Mayer agradece à CAPES pela bolsa de mestrado. Luiz H. A. Monteiro agradece ao CNPq pela bolsa de produtividade. Ambos agradecem a Alex Martins pelo suporte computacional no início deste trabalho.

\section{Referências}

[1] J. S. Bloom and G. W. Hynd, The role of the corpus callosum in interhemispheric transfer of information: excitation or inhibition?, Neuropsychol. Rev., vol. 15, 59$71(2005)$.

[2] T. Burwick, The binding problem, WIREs Cogn. Sci., vol. 5, 305-315 (2014).

[3] M. Castelo-Branco, S. Neuenschwander and W. Singer, Synchronization of visual responses between the cortex, lateral geniculate nucleus, and retina in the anesthetized cat, J. Neurosci., vol. 18, 6395-6410 (1998).

[4] T. Gollisch, Throwing a glance at the neural code: rapid information transmission in the visual system, HFSP J., vol. 3, 36-46 (2009).

[5] H. Ito, P. E. Maldonado and C. M. Gray, Dynamics of stimulus-evoked spike timing correlations in the cat lateral geniculate nucleus, J. Neurophysiol., vol. 104, 3276-3292 (2010).

[6] A. Martins and L. H. A. Monteiro, Frequency transitions in synchronized neural networks, Commun. Nonlinear Sci. Numer. Simulat., vol. 18, 1786-1791 (2013).

[7] L. H. A. Monteiro, Sistemas Dinâmicos Complexos, Livraria da Física (2014).

[8] M. E. J. Newman and D. J. Watts, Renormalization group analysis of the smallworld network model, Phys. Lett. A, vol. 263, 341-346 (1999).

[9] M. Ozer, M. Perc and M. Uzuntarla, Stochastic resonance on Newman-Watts networks of Hodgkin-Huxley neurons with local periodic driving, Phys. Lett. A, vol. 373, 964968 (2009).

[10] S. H. Strogatz, Sync, Hyperion (2003).

[11] P. J. Uhlhaas and W. Singer, Neural synchrony in brain disorders: relevance for cognitive dysfunctions and pathophysiology, Neuron, vol. 52, 155-168 (2006). 